\title{
Involvement of the Transcriptional Coactivator ThMBF1 in the Biocontrol Activity of Trichoderma harzianum
}

\section{Belén Rubio ${ }^{1}$, Alonso J. Pardal', Rosa E. Cardoza ${ }^{2}$, Santiago Gutiérrez², Enrique Monte ${ }^{1}$ and Rosa Hermosa ${ }^{1 *}$}

${ }^{1}$ Spanish-Portuguese Institute for Agricultural Research (CIALE), Department of Microbiology and Genetics, University of Salamanca, Salamanca, Spain, ${ }^{2}$ Area of Microbiology, University School of Agricultural Engineers, University of León, Ponferrada, Spain

Trichoderma harzianum is a filamentous fungus well adapted to different ecological niches. Owing to its ability to antagonize a wide range of plant pathogens, it is used as a biological control agent in agriculture. Selected strains of T. harzianum are also able to increase the tolerance of plants to biotic and abiotic stresses. However, little is known about the regulatory elements of the T. harzianum transcriptional machinery and their role in the biocontrol by this species. We had previously reported the involvement of the transcription factor THCTF1 in the T. harzianum production of the secondary metabolite 6-pentyl-pyrone, an important volatile compound related to interspecies cross-talk. Here, we performed a subtractive hybridization to explore the genes regulated by THCTF1, allowing us to identify a multiprotein bridging factor 1 (mbf1) homolog. The gene from T. harzianum T34 was isolated and characterized, and the generated Thmbf1 overexpressing transformants were used to investigate the role of this gene in the biocontrol abilities of the fungus against two plant pathogens. The transformants showed a reduced antifungal activity against Fusarium oxysporum f. sp. lycopersici race $2(\mathrm{FO})$ and Botrytis cinerea $(\mathrm{BC})$ in confrontation assays on discontinuous medium, indicating that the Thmbf1 gene could affect T. harzianum production of volatile organic compounds (VOC) with antifungal activity. Moreover, cellophane and dialysis membrane assays indicated that Thmbf1 overexpression affected the production of low molecular weight secreted compounds with antifungal activity against FO. Intriguingly, no correlation in the expression profiles, either in rich or minimal medium, was observed between Thmbf1 and the master regulator gene cross-pathway control (cpc1). Greenhouse assays allowed us to evaluate the biocontrol potential of $T$. harzianum strains against $\mathrm{BC}$ and $\mathrm{FO}$ on susceptible tomato plants. The wild type strain T34 significantly reduced the necrotic leaf lesions caused by BC while plants treated with the Thmbf1-overexpressing transformants exhibited an increased susceptibility to this pathogen. The percentages of Fusarium wilt disease incidence and values of aboveground dry weight showed that T34 did not have biocontrol activity against FO, at least in the 'Moneymaker' tomato variety, and that Thmbf1 overexpression increased the incidence of this disease. Our results show that the Thmbf1 overexpression in T34 negatively affects its biocontrol mechanisms.

Keywords: biological control, antifungal activity, multiprotein bridging factor, volatile organic compounds, Fusarium oxysporum f. sp. lycopersici 


\section{INTRODUCTION}

Trichoderma is a genus of filamentous fungi distributed worldwide, extremely well suited to live in different ecological niches (Druzhinina et al., 2011). This is due to its remarkably diverse metabolism, capable of catabolising a broad variety of substrates as well as of producing a huge diversity of secondary metabolites (Mukherjee et al., 2013). Trichoderma (where known, the teleomorphs belong to Hypocrea) includes species currently used as biological control agents due to their ability to antagonize a wide range of plant pathogens (Harman et al., 2004), Trichoderma harzianum being one of the species most widely used in biocontrol (Monte, 2001; Lorito et al., 2010). Selected Trichoderma rhizosphere-competent strains have been shown to exert beneficial effects on plants, increasing growth and stimulating defences against biotic and abiotic damage (Shoresh et al., 2010; Hermosa et al., 2012; Rubio et al., 2017).

Transcriptional coactivators play a crucial role in eukaryotic gene expression by connecting TATA-binding proteins (TBP) and the associated basal transcription machinery to transcription factors (TFs) (Suzuki et al., 2005). Some TFs have been functionally characterized in Trichoderma spp. (Aro et al., 2003; Casas-Flores et al., 2004; Stricker et al., 2006; Rubio et al., 2009; Fu et al., 2012; Gruber and Zeilinger, 2014, among others). However, little is known about other regulatory elements of the Trichoderma spp. transcriptional machinery and their role in biocontrol. Members of the highly conserved multiprotein bridging factor 1 (MBF1) protein family function as non-DNAbinding transcriptional coactivators. These mediator proteins are involved in regulating metabolic and developmental pathways in different organisms ranging from fungi to animals ( $\mathrm{Li}$ et al., 1994; Takemaru et al., 1997). It has been demonstrated that MBF1 proteins interact with TFs or with different hormone receptors and link them with TBP, as observed in yeasts (Takemaru et al., 1998), fruit flies (Liu et al., 2003) or humans (Brendel et al., 2002; Kabe et al., 2005). MBF1 is also crucial for response to oxidative stress in human cells (Miotto and Struhl, 2006). In plants, MBF1 of Arabidopsis thaliana is encoded by three genes: $M b f 1 a$ and $M B f 1 b$, which are regulated developmentally (Tsuda et al., 2004), and Mbf1c that was related to expression changes of 36 transcripts during heat-stress (Suzuki et al., 2008). The potato MBF1 protein is induced in response to attack by a pathogen (Godoy et al., 2001) as well as to heat and oxidative stresses (Arce et al., 2006).

Most studies addressing fungal MBF1 have been carried out in yeasts. This coactivator mediates the general control non-derepressible (GCN4) protein-dependent transcriptional activation in Saccharomyces cerevisiae (Takemaru et al., 1998). GCN4 is a TF controlled at multiple levels by diverse signals of starvation and stress. This master regulator of gene expression acts modulating the transcription of amino acid biosynthesis genes, among others (Hinnebusch and Natarajan, 2002). In filamentous fungi, the cross-pathway control 1 gene, cpc1, encodes a protein similar to the yeast GCN4 (Paluh et al., 1988).

Little has been reported about MBF1 in filamentous fungi. In Fusarium fujikuroi, deletion of the areA gene, encoding a TF that mediates nitrogen metabolite repression, leads to an upregulation of amino acid biosynthesis genes as well as $c p c 1$ and its putative co-regulator $m b f 1$, both under nitrogen starvation and abundance (Schönig et al., 2008). However, $\Delta m b f 1$ mutants of F. fujikuroi do not show differences in gene expression regulated by the factor CPC1 (Schönig et al., 2009). Thus, CPC1 mediates cross-pathway control independently of MBF1, at least in this fungus (Schönig et al., 2009). Furthermore, it has been identified and characterized an mbf1 homolog in Beauveria bassiana and Magnaporthe oryzae as being involved in hyphal growth and stress responses (Ying et al., 2014; Fan et al., 2017). In addition, it has been demonstrated that the lack of $B b M B F 1$ in $B$. bassiana reduced its pathogenicity level against Galleria mellonella larvae (Ying et al., 2014), and that MoMBF1 contributes to the virulence of $M$. oryzae in rice plants (Fan et al., 2017).

In a previous study working with Thctf1-null mutants from T. harzianum T34, we demonstrated that the TF THCTF1 was related to the biosynthesis of 6-pentyl-2H-pyran-2-one (6PP) derivatives and biocontrol activity in this fungus (Rubio et al., 2009). Here, an mbf1 homolog was identified in a suppression subtractive hybridization between the wild type strain T34 and a Thetf1-null mutant. The aim of this study was to functionally characterize $m b f 1$ in $T$. harzianum. We overexpressed the Thmbf1 gene in strain T34 and studied its involvement in the antagonistic activity of T34 against Fusarium oxysporum f. sp. lycopersici and Botrytis cinerea in in vitro assays, and in the biocontrol potential against these two pathogens on tomato plants in greenhouse assays. Expression levels of Thmbf1 and $c p c 1$ genes in T. harzianum strains grown on rich and minimal media were analyzed to investigate whether both genes might be functionally related.

\section{MATERIALS AND METHODS}

\section{Bacterial and Fungal Strains and Tomato Seeds}

Escherichia coli $\mathrm{DH} 5 \alpha$ was used as a host for plasmid construction and propagation. This bacterial strain was grown in Luria-Bertani (LB) broth or on LB agar dishes, supplemented with ampicillin (100 $\mu \mathrm{g} / \mathrm{ml})$, X-gal $(40 \mu \mathrm{g} / \mathrm{ml})$ and IPTG $(10 \mu \mathrm{g} / \mathrm{ml})$, when required.

Trichoderma harzianum T34 (CECT 2413, Spanish Type Culture Collection, Valencia, Spain) was used as a source of DNA to clone the Thmbf1 gene and also as a host in the transformation experiments to overexpress the Thmbf 1 gene. Fusarium oxysporum f. sp. lycopersici strain 4287 (FO), determined as race 2 (Di Pietro and Roncero, 1998) and Botrytis cinerea B05.10 (BC), were used as plant pathogenic microorganisms in in vitro and in vivo assays. Fungal strains were routinely grown on PDA (Difco Becton Dickinson, Sparks, MD), and conidia were stored at $-80^{\circ} \mathrm{C}$ in $30 \%$ glycerol.

Tomato (Solanum lycopersicum) seeds of 'Moneymaker' (Dobies \& Paignton, Devon, United Kingdom) and 'Marmande' (Thompson \& Morgan, Ipswich, United Kingdom) varieties were 
used in greenhouse assays. Seeds were superficially disinfected in $70 \%$ ethanol for $10 \mathrm{~min}$ and in $2 \%$ sodium hypochlorite for $10 \mathrm{~min}$. Later on, they were rinsed thoroughly three times in sterile distilled water before use, and air-dried on a sterile gauze sheet.

\section{Selection and Isolation of Thmbf1}

A suppression subtractive hybridization (SSH) between cDNAs from T. harzianum strains T34 and $\Delta \mathrm{D} 1-38$, a $\Delta$ Thctf1 mutant affected in the production of 6-PP derivatives (Rubio et al., 2009), was carried out. Mycelia from both strains were obtained after growth under identical conditions as follows: $100 \mathrm{ml}$ of $\mathrm{CM}$ medium $(0.5 \%$ malt extract, $0.5 \%$ yeast extract, $0.5 \%$ glucose $)$ was inoculated with $10^{8}$ conidia from PDA cultures, and incubated at $28^{\circ} \mathrm{C}$ in an orbital incubator at $250 \mathrm{rpm}$ for $20 \mathrm{~h}$. Then, $18 \mathrm{ml}$ of the CM culture was inoculated into a Roux flask, containing $250 \mathrm{ml}$ of $\mathrm{MM}$ containing $0.5 \%$ glucose (Penttilä et al., 1987). The culture was incubated statically at $28^{\circ} \mathrm{C}$ for 7 days in a growth chamber with a $12 \mathrm{~h}$ light/12 h dark photoperiod. Trichoderma mycelia were harvested by filtration through nytal (30- $\mu \mathrm{m}$ pore diameter), and RNA was extracted as previously described (Cardoza et al., 2006b). The mRNA was purified by oligo (dT) cellulose columns (Stratagene, La Jolla, CA, United States). Five microgram of mRNA were used for cDNA synthesis, using a cDNA synthesis system (Roche Diagnostics, Mannheim, Germany) and following the manufacturer's instructions.

The cDNAs from both strains were used for a subtractive hybridization using the PCR-Select cDNA subtraction kit (Clontech laboratories, Palo Alto, CA, United States) (Diatchenko et al., 1996), following the manufacturer protocol.

The complete sequence of the Thmbf1 gene was obtained from a screening of a T. harzianum T34 lambda genomic library (Lora et al., 1995) as previously described (Rubio et al., 2009). DNAbinding elements were found by looking for consensus sequences described elsewhere or by using the MatInspector program ${ }^{1}$ with the TRANSFAC database restricted to fungi.

\section{Conventional PCR Amplification and Sequencing}

PCR amplifications were accomplished using the Taq polymerase system (Biotools, Edmonton, AB, Canada), following the manufacturer's instructions. The Thmbf1 cDNA was PCR-amplified with the primers MBF1-5 (5'- ATGTCTAACCAGGACTGGGA TT-3') and MBF1-3 (5'-TTATTTCTTCTTGGGGCCCAAG-3') and T34 cDNA as template. Screening of T. harzianum T34 Thmbf1 overexpressing transformants was performed by PCR with the primers Phleo-3 (5'-GGTGTTGGTCGGCGTCGG-3') and GPD-3 (5'-GGTGTGTCGGCGGGGTTG-3') to amplify a 645-bp fragment from the $\mathrm{p} 43 \mathrm{~b} 1 \mathrm{MBFa}$ plasmid.

The PCR products were purified from agarose gels using the NucleoSpin Extract II Kit (Macherey-Nagel) according to the manufacturer's protocol. PCR fragments were sequenced and the sequences were analyzed using the DNASTAR package (Lasergene, Madison, WI, United States).

\footnotetext{
${ }^{1}$ http://www.genomatix.de/matinspector.html
}

\section{Plasmid Constructions and Trichoderma Transformation Procedure}

Plasmid p43b1MBFa was used for the transformation. To construct it, plasmid pAN52.1 (Punt et al., 1987), which contained the $g p d A$ (glyceraldehyde-3-phosphate dehydrogenase) gene promoter and the $\operatorname{trp} C$ gene terminator from Aspergillus nidulans, was digested with NcoI, treated with Klenow fragment and dephosphorylated with calf intestine alkaline phosphatase (CIAP). Then, it was ligated to the Thmbf1 cDNA, which was amplified using the oligonucleotides MBF15 and MBF1-3. As result, the pAN52.1-MBF1a (6367 bp) plasmid was obtained. This plasmid was PstI-digested, treated with Klenow fragment, and the resulting 3571 bp fragment, containing the Thmbf1 expression cassette, was gel-purified. This fragment was ligated to the pJL43b1 plasmid (Gutiérrez et al., 1997), which contained the ble gene from Streptoalloteichus hindustanus under the control of the gpdA gene promoter, previously digested with $K p n \mathrm{I}$, treated with Klenow fragment, and CIAP-dephosphorylated. The resulting plasmid, p43b1MBFa (8067 bp), was used to transform protoplasts of the T34 strain (Cardoza et al., 2006a). In parallel, strain T34 was also transformed with pJL43b1 to obtain empty vector transformants; one of them was included in assays as a transformation control. Transformants were selected for phleomycin resistance.

\section{Hybridization Experiments}

For Southern blot analysis, total DNA was extracted as previously described (Cardoza et al., 2006a). Then, $10 \mu \mathrm{g}$ of genomic DNA was XhoI- and BamHI-digested, electrophoresed on a $0.7 \%$ agarose gel, and transferred to a Hybond- $\mathrm{N}^{+}$membrane (Amersham, Piscaway, NJ, United States). The Thmbf1 cDNA gene was labeled using the DIG High Prime kit (Roche, Penzberg, Germany), following the manufacturer's instructions, and used as a probe. Hybridization, washes and detection were carried out as previously described (Tijerino et al., 2011).

\section{Phenotypic Assays}

The growth of the wild type, transformation control and transformant strains was tested under different culture media. Two hundred conidia of each strain were used to inoculate dishes containing PDA or minimal medium (Penttilä et al., 1987) and incubated at $28^{\circ} \mathrm{C}$ for 3 days. These assays were performed in triplicate.

Mycelia from the transformation control Thmbf-CT and transformants were collected from both culture conditions and used to analyze the expression levels of Thmbfland $c p c 1$ genes

\section{In Vitro Antifungal Assays Dual Confrontations}

In vitro confrontation assays between Trichoderma strains and the pathogens $\mathrm{FO}$ and $\mathrm{BC}$ were carried out on PDA at $28^{\circ} \mathrm{C}$ as previously described (Rubio et al., 2009) and photographs were taken after 10 days. These assays were performed in triplicate, and single cultures of Trichoderma strains and pathogens were used as controls. 


\section{Confrontations on Discontinuous Medium}

Strains of $T$. harzianum were also confronted with pathogens FO and BC on PDA using 90-mm Petri dishes separated in two halves. Trichoderma and pathogen were inoculated to their respective half. Cultures were incubated at $28^{\circ} \mathrm{C}$ in the dark, and colony diameter measures and photographs were taken 5 days after inoculation. Pathogen cultures grown alone were used as controls.

\section{Growth Assays on Membranes}

Five-mm-diameter PDA plugs of $T$. harzianum T34, transformation control or transformants were placed, at the center of Petri dishes containing PDA, on cellophane sheets or on $14 \mathrm{kDa}$-cut-off dialysis membranes. After 2 days of incubation at $28^{\circ} \mathrm{C}$, the membranes were removed from the dishes and a single $5-\mathrm{mm}$ diameter mycelial plug of FO or BC was placed at the center of each dish. In parallel, each pathogen was grown on PDA (control dishes). Each condition was tested in triplicate and the results were expressed as growth diameters of each pathogen after incubation for 2 days on PDA.

\section{Real-Time Quantitative PCR}

Gene expression was analyzed by real-time quantitative PCR (qPCR). cDNAs were synthesized from $1 \mu \mathrm{g}$ of total RNA, using the Transcriptor First Strand cDNA Synthesis kit (Takara Inc., Tokyo, Japan) with an oligo(dT) primer. Reaction mixtures and amplification conditions were performed as previously described (Montero-Barrientos et al., 2010). PCRs were carried out in triplicate for three different biological replicates. Data are expressed using the $2^{-\Delta \Delta C_{\mathrm{T}}}$ method (Livak and Schmittgen, 2001). The following primer pairs were used and checked for dimer formation: 414 (5'-CTCAGCTTGACGTTG ACGAC- $\left.3^{\prime}\right)$ and 415 (5'-CTACACCCGACCAGACCATT- $\left.3^{\prime}\right)$, Cpc1-bf (5'-CGTCGATTTGGACGACTTCAC-3') and Cpc-br (5'-GAGGAGACACGGTGCCAAGATT- $3^{\prime}$ ), and Act-1 (5'-ATC GGTATGGGTCAGAAGGA-3') and Act-2 (5'-ATGTCAACAC GAGCAATGG-3'), amplifying fragments of the Thmbf1, cpc1 and actin Trichoderma genes, respectively. The primer pair $\mathrm{Cpc}-$ bf and Cpc-br was designed using a sequence alignment of the $c p c 1$ gene identified in the annotated genomes of T. atroviride, $T$. reesei and $T$. virens. Standard curves were measured for dilution series of pooled cDNA samples, and calculated using Applied Biosystems software.

\section{Biocontrol Assays in Tomato Plants}

The biocontrol ability of four $T$. harzianum strains (T34, Thmbf-CT, Thmbf-ov3 and Thmbf-ov4) against FO and BC on susceptible tomato plants was evaluated in in vivo assays.

\section{FO Assays}

Two independent assays were performed only differing in the method of inoculation with the $T$. harzianum strains: 'Moneymaker' tomato seed or substrate applications. In the first assay, surface-sterilized seeds as described above were coated with $1 \mathrm{ml}$ of an aqueous suspension containing $1 \times 10^{8}$ conidia per $\mathrm{ml}$ or with $1 \mathrm{ml}$ of sterile water (control) as previously described (Pérez et al., 2015). One $\mathrm{ml}$ was used to coat 30 seeds. Coated seeds were sowed in multi-cell growing trays containing a mixture of commercial substrate (Projar Professional-Comercial Projar, Valencia, Spain) and vermiculite (3:1), previously autoclaved for $1 \mathrm{~h}$ at $121^{\circ} \mathrm{C}$ on two successive days. In the second assay, surface-sterilized seeds were sown in $0.7-1$ pots (one seed per pot) containing $200 \mathrm{~g}$ of the above described autoclaved commercial substrate inoculated with T. harzianum ( $10^{8}$ conidia per pot). In both assays, seedlings were maintained under greenhouse conditions at $22 \pm 4^{\circ} \mathrm{C}$ and a photoperiod of $16 \mathrm{~h}$ light: $8 \mathrm{~h}$ dark. Fourteen days after sowing, when the first true leaf was fully expanded, seedlings were uprooted, the excess of peat removed by shaking and roots cut to about $2.5 \mathrm{~cm}$. The cut-root seedlings were dipped in a FO conidial suspension, adjusted to $2 \times 10^{7}$ conidia per $\mathrm{ml}$, and planted in $0.7-1$ pots containing the above indicated mixture. FO conidia were obtained from 7 days-PDB cultures. Ten pots per treatment and one seedling per pot were used for each assay. Seedlings dipped with sterile water were included as a control. After FO inoculation, seedlings were maintained in the greenhouse under the conditions described above for 3 weeks, and watered as needed.

The six treatments tested were as follows: untreated (control), FO, T34 + FO, Thmbf-CT + FO, Thmbf-ov3 + FO, and Thmbfov $4+$ FO. In both assays, ten plants were used per treatment in a completely randomized design. The disease index was calculated using the following symptom severity scale (0-4): 0 , healthy plant; 1,2 , and 3 , slight, moderate and severe wilting plant, respectively; and 4, dead plant; and values used to determine the disease incidence (DI) percentage, as previously described (Song et al., 2004). Aboveground dry weights were also recorded for the assay T. harzianum treated-seeds.

\section{Assay of BC}

Surface-sterilized 'Marmande' tomato seeds were coated with an aqueous suspension of T. harzianum conidia or water (control) as described above. Seeds were sowed in $0.7-1$ pots (one seed per pot) containing the autoclaved mixture above indicated and seedlings were maintained under the indicated greenhouse conditions for 4 weeks. The sensitivity of plants to BC was evaluated as previously described (Pérez et al., 2015), except that two leaves from each plant were inoculated in a single point. Necrotic leaf area was evaluated after 3 days using ImageJ free software. Five plants were considered for each of the six treatments tested: untreated (control), BC, T34 + BC, ThmbfCT + BC, Thmbf-ov3 + BC and Thmbf-ov4 + BC.

\section{Statistical Analyses}

Each data set was submitted to analysis of variance (ANOVA) and means compared by Tukey test $(\mathrm{P}<0.05)$ using Statistica 7 software (Statsoft Inc., Palo Alto, CA, United States).

\section{RESULTS}

\section{The T. harzianum T34 Thmbf1 Gene}

A SSH method was carried out with cDNAs from the wild type strain T. harzianum T34 and the $\Delta \mathrm{D} 1-38$ knock-out mutant. 
$\Delta$ D1-38 had been previously used to explore 6-PP biosynthesisrelated genes regulated by the TF THCTF1 (Rubio et al., 2009). A total of 202 differentially expressed clones were isolated, sequenced and analyzed using BlastX software. As a result, 96 clones showed homology with known genes (Supplementary Table S1). Six of them (6.5\% of the total identified clones) corresponded to an $m b f 1$ homolog, which was selected for further characterization. The 340-bp fragment isolated from the T. harzianum subtractive library was used as a probe to screen a lambda genomic library. A total of 657 bp containing the complete open reading frame (ORF) of Thbmf1 and 119 bp of the promoter region were sequenced from a positive phage. Thmbf1 has a length of $538 \mathrm{bp}$ and contains one intron of $70 \mathrm{bp}$. The ORF, excluding the intron, contains $468 \mathrm{bp}$ and encodes a protein of 156 amino acids with a theoretical molecular mass of $16.4 \mathrm{kDa}$ and an isoelectric point of 10.2. The nucleotide sequence of Thmbf1 was deposited in the GenBank database under Accession No. CCG26107. One single copy of Thmbf1 homolog was detected in publicly available Trichoderma spp. genomes such as $T$. reesei (94\% protein identity, ID 122457 protein), T. virens (94\% protein identity, ID 73623 protein) and T. atroviride (90\% protein identity, ID 151694 protein). Analysis of the 156 amino acids of the predicted T. harzianum T34 ThMBF1 protein revealed the presence of one DNAbinding helix-turn-helix (HTH) domain (amino acids 81-117), as described previously for eukaryotes (Aravind and Koonin, 1999), and the prevalence of the alpha-helix conformation. A high degree of similarity (70\% amino acid sequence identity) was also found with the MBF1 proteins from fungi such as BbMBF1 of B. bassiana (XP008595149).

\section{Overexpression of Thmbf1 in T. harzianum T34}

In order to functionally characterize the Thmbf1 gene, the plasmid p43b1MBF1a was constructed and transformed in T. harzianum T34. Thirty transformants showing phleomycin resistance were checked by PCR. A 645-bp PCR product was amplified in nine of the thirty transformants analyzed, using the primer pair Phleo-3 and GPD-3. Four PCR-positive putative transformants were randomly chosen for further analysis by Southern blot (Thmbf-ov1, Thmbf-ov2, Thmbf-ov3 and Thmbfov4) and determination of the additional Thmbf1 copies due to the insertion of the transformation cassette in their genomes (Supplementary Figure S1). DNAs from strains T34 and ThmbfCT, an empty vector transformant, were included as controls. One $0.8 \mathrm{~kb}$ signal, which corresponded to the endogenous gene, was observed in all lanes, indicating that Thmbf1 is present as a single copy in T. harzianum T34 and that the transformation cassette is not present in strain Thmbf-ov1. Several blotted bands corresponding to the Thmbf1 gene were observed in DNAs from three out of four transformant strains analyzed, indicating that the transformation cassette had been inserted into the Thmbf-ov2, Thmbf-ov3 and Thmbf-ov4 genomes at several loci.

Additional PCR reactions were carried out with DNA from the strain Thmbf-ov1 and the primer pairs MBF1-5 and MBF1-3 and
Pleo-3 and GPD-3. A 558-bp PCR fragment was amplified with the pair MBF1-5 and MBF1-3, but no PCR product was observed when Pleo-3 and GPD-3 primers were used. Moreover, strain Thmbf-ov1 lost its ability to grow on PDA containing $100 \mu \mathrm{g} / \mathrm{mL}$ of phleomycin.

\section{Thmbf1 and cpc1 Expression Patterns under Different Culture Conditions}

We analyzed the expression level of the Thmbf1 gene by qPCR with the primer pairs $414 \& 415$ and Act-1\&Act- 2 in the Thmbf-ov1, Thmbf-ov2, Thmbf-ov3 and Thmbf-ov4 strains after growing on PDA and minimal media using the expression level in strain Thmbf-CT as a reference condition. The calibration slope, $R^{2}$ and efficiency of these primer pairs were: - 3.26, 0.95 and $114.55 \%$, for 414 and 415, and - 3.38, 0.95 and $96.61 \%$, for Act- 1 and Act-2. Transformants Thmbf-ov2, Thmbf-ov3 and Thmbfov4 showed higher Thmbf1 transcript levels than those observed for Thmbf-CT after growing on both media (Figure 1A), whereas no differences were detected between Thmbf-ov1 and Thmbf-CT strains.

In order to identify the influence of Thmbf1 on the transcription of $c p c 1$, the transcript levels of this latter were also examined in strains Thmbf-CT, Thmbf-ov1, Thmbf-ov2, Thmbf-ov3 and Thmbf-ov4 after growing under identical culture conditions. We used the primer pairs $\mathrm{Cpc}-\mathrm{Bf}$ and $\mathrm{Cpc}-\mathrm{Br}$, which had values of $-3.13,0.99$, and $108.73 \%$ for calibration slope, $R^{2}$ and efficiency, respectively, and Act-1 and Act2. No significant expression differences were observed among the five tested strains (Figure 1B).

\section{Antagonistic Activity}

Dual confrontations assays between T34, Thmbf-CT, Thmbfov1, Thmbf-ov2, Thmbf-ov3 and Thmbf-ov4, and the pathogens FO or BC were performed to investigate the effect of Thmbf1 overexpression on the antagonistic activity of T. harzianum T34. All the assayed $T$. harzianum strains inhibited the growth of both pathogens on PDA, although they were not able to grow over the colonies of FO and BC (Supplementary Figure S2). No different behavior was observed among the wild type or the transformation control and the strains Thmbf-ov1, Thmbf-ov3 and Thmbf-ov4, whereas less ability to inhibit colony growth of FO and BC was observed for the Thmbf-ov2 strain. On PDA and minimal media, the Thmbf-ov2 strain displayed a smaller growth phenotype than the other strains, whereas no differences were observed between the rest of them (data not shown). At this stage, transformants Thmbf-ov3 and Thmbf-ov4 were selected for further analyses.

In order to analyze whether Thmbf1 is involved in the production of volatile organic compounds (VOC) with antifungal activity, dual confrontations between T. harzianum strains T34, Thmbf-CT, Thmbf-ov3 and Thmbf-ov4, and the pathogens FO or $\mathrm{BC}$ were carried out on PDA discontinuous medium. The antifungal activity due to hydrolases and metabolites released to the culture medium was avoided since each fungus grew in different halves of the Petri dish. Strains Thmbf-ov3 and Thmbfov4 differed significantly in their ability to inhibit colony growth 

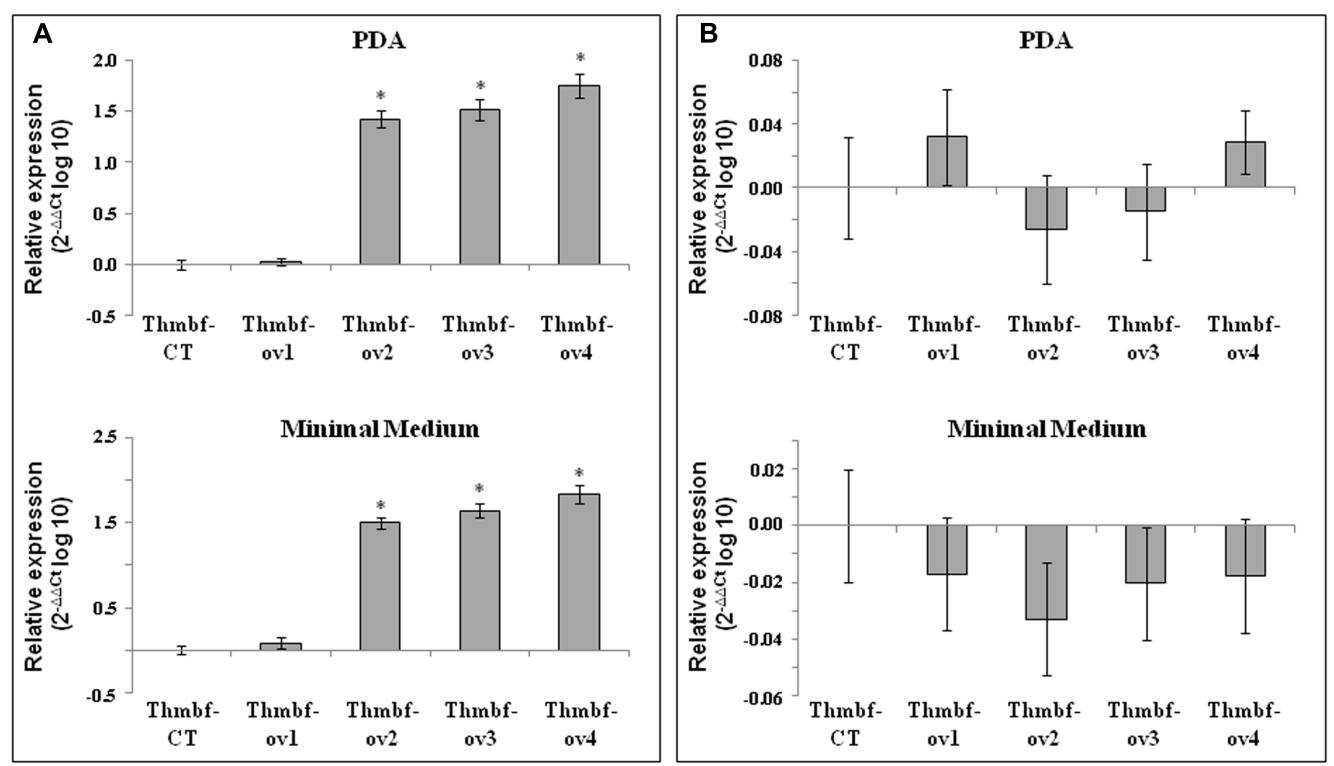

FIGURE 1 | Transcript levels of Thmbf1 (A) and cpc1 (B) in four putative Thmbf1 overexpressed transformants -T. harzianum Thmbf-ov1, Thmbf-ov2, Thmbf-ov3 and Thmbf-ov4- by qPCR. Values correspond to relative measurements against the Thmbf1 or cpc1 transcripts in the control transformant T. harzianum Thmbf-CT $\left(2^{-\Delta \Delta C_{t}}=1\right)$, and are expressed as $\log _{10}$. The experiment was carried out with mycelia grown at $28^{\circ} \mathrm{C}$ for 3 days on PDA and minimal media. T. harzianum actin was used as an internal reference gene. Bars represent the standard deviations of the mean of three replicates. Asterisk $\left({ }^{*}\right)$ represents statistically significant differences $(P<0.05)$.

TABLE 1 | Growth of F. oxysporum f. sp. lycopersici (FO) and B. cinerea (BC) confronted with T. harzianum strains on discontinuous medium.

Colony diameter $(\mathrm{cm})$

\begin{tabular}{lcc}
\cline { 2 - 3 } Thesis & FO & BC \\
\hline Control & $4.15 \pm 0.15 b$ & $3.52 \pm 0.03 \mathrm{a}$ \\
T34 & $3.15 \pm 0.05 \mathrm{c}$ & $1.10 \pm 0.13 \mathrm{c}$ \\
Thmbf-CT & $2.90 \pm 0.10 \mathrm{c}$ & $1.25 \pm 0.05 \mathrm{c}$ \\
Thmbf-ov3 & $3.95 \pm 0.25 \mathrm{~b}$ & $1.45 \pm 0.05 \mathrm{~b}$ \\
Thmbf-ov4 & $4.60 \pm 0.20 \mathrm{a}$ & $1.62 \pm 0.03 \mathrm{~b}$
\end{tabular}

Trichoderma harzianum strains correspond to the wild-type (T34), the transformation control (Thmbf-CT) and the Thmbf1 overexpressing transformants (Thmbf-ov3 and Thmbf-ov4). Colony diameters (cm) were measured after 5 days growing on discontinuous PDA medium at $28^{\circ} \mathrm{C}$. Values are means of three replicates with the corresponding standard deviations. For each column, values followed by different letter are significantly different $(P<0.05)$.

of FO and BC (Table 1), showing less antifungal activity than T34 or Thmbf-CT (Figure 2). Particularly, Thmbf1 overexpressing transformants did not reduce the colony size of FO when they were tested in discontinuous medium.

To examine the role of the Thmbfl gene in the secretion of different molecular weight (MW) compounds with antifungal activity in T. harzianum, assays were performed with cellophane (allowing small and large compounds to pass through) and dialysis membranes with a MW cut-off of $14 \mathrm{kDa}$ (allowing only metabolites $<14 \mathrm{kDa}$ to pass through). Table 2 summarizes the colony diameters of $\mathrm{FO}$ and $\mathrm{BC}$ after growing on PDA medium containing Trichoderma extracellular compounds. All T. harzianum strains assayed were able to inhibit the growth of both pathogens. No differences were detected between both types of membranes, indicating that small compounds secreted by $T$. harzianum are major contributors to the inhibitory activity observed against FO and BC. Moreover, significantly lower FO growth inhibition was recorded for strains Thmbfov3 and Thmbf-ov4 compared to that of T34 and ThmbfCT on both cellophane and dialysis membranes. However, no significant BC growth inhibition differences were observed among the four $T$. harzianum strains on both types of membranes.

\section{Effect of Overexpressing Thmbf1 Gene on T. harzianum Biocontrol Capability Against Botrytis Leaf Lesions in Tomato Plants}

Four-week-old 'Marmande' tomato plants previously seedcoated with an aqueous solution (control) or treated with conidia of T34, Thmbf-CT, Thmbf-ov3 or Thmbf-ov4 were leaf inoculated with BC. Necrotic spots were observed 3 days after inoculation of $\mathrm{BC}$ whereas no lesions were detected in BC-uninoculated plants, results are shown in Figure 3. The lowest necrotic leaf area was observed in plants of the T34 and Thmbf-CT treatments, and no significant statistically differences were detected between them. However, plants treated with Thmbf1-overexpressing transformants showed the highest lesion sizes, being similar to those observed in the control plants. These results indicate that T34 is able to control BC in 'Marmande' plants and the overexpression of Thmbf 1 gene in this strain reduces its biocontrol ability against the pathogen $\mathrm{BC}$. 


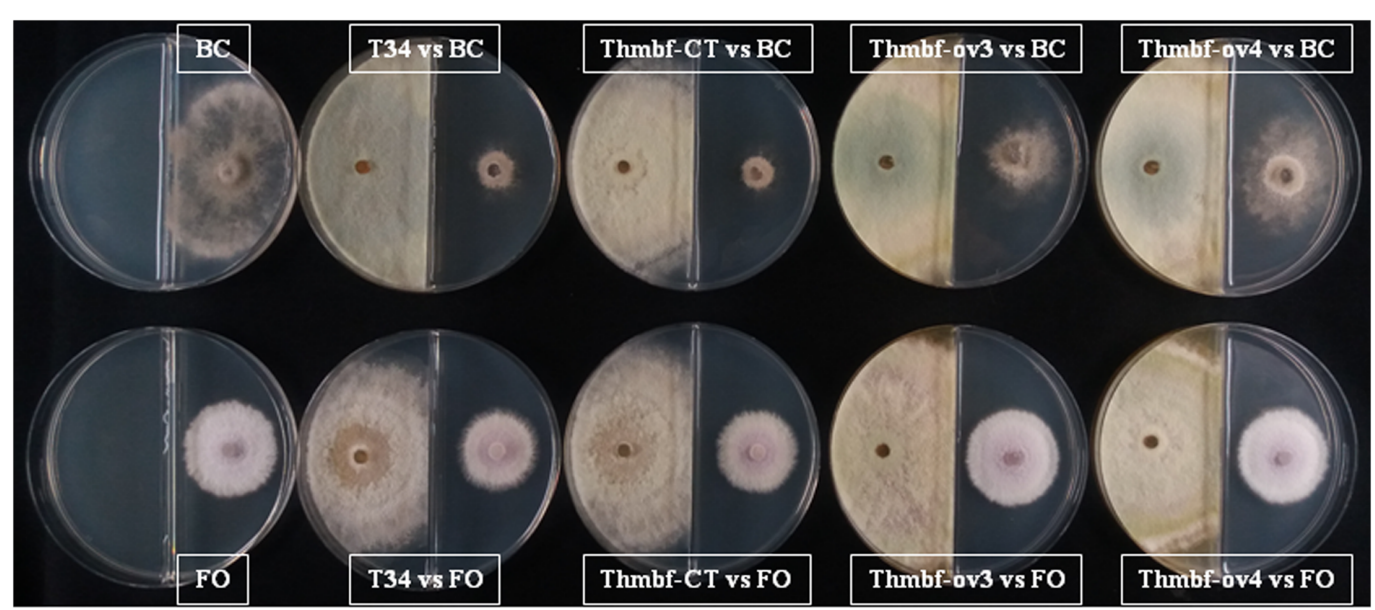

FIGURE 2 | Dual cultures of strains T34, Thmbf-CT, Thmbf-ov2, Thmbf-ov3 and Thmbf-ov4 of T. harzianum and the pathogens B. cinerea (BC) and F. oxyxporum (FO) on discontinuous PDA medium. Plates only with the pathogen were used as controls. All plates were incubated at $28^{\circ} \mathrm{C}$ for 5 days.

TABLE 2 | Growth of F. oxysporum f. sp. lycopersici (FO) and B. cinerea (BC) on PDA medium, where T. harzianum wild-type (T34), transformation control (Thmbf-CT) or Thmbf1 overexpressing transformants (Thmbf-ov3 and Thmbf-ov4) strains were previously grown on cellulose (cut-off $14 \mathrm{kDa}$ ) or cellophane membranes for 2 days at $28^{\circ} \mathrm{C}$.

\section{Colony diameter $(\mathrm{cm})$}

\begin{tabular}{llllll}
\cline { 2 - 3 } Thesis & \multicolumn{2}{c}{ Fo } & & \multicolumn{2}{c}{ BC } \\
\cline { 2 - 3 } \cline { 5 - 6 } & Cellophane & Cellulose & & Cellophane & Cellulose \\
\hline Control & $2.33 \pm 0.05 \mathrm{a}$ & $2.33 \pm 0.05 \mathrm{a}$ & & $2.75 \pm 0.18 \mathrm{a}$ & $2.75 \pm 0.18 \mathrm{a}$ \\
T34 & $0.50 \pm 0.00 \mathrm{c}$ & $0.50 \pm 0.00 \mathrm{~d}$ & & $0.57 \pm 0.10 \mathrm{~b}$ & $0.63 \pm 0.10 \mathrm{~b}$ \\
Thmbf-CT & $0.50 \pm 0.00 \mathrm{c}$ & $0.50 \pm 0.00 \mathrm{~d}$ & & $0.58 \pm 0.13 \mathrm{~b}$ & $0.65 \pm 0.23 \mathrm{~b}$ \\
Thmbf-ov3 & $0.67 \pm 0.05 \mathrm{~b}$ & $0.63 \pm 0.10 \mathrm{c}$ & & $0.50 \pm 0.00 \mathrm{~b}$ & $0.50 \pm 0.00 \mathrm{~b}$ \\
Thmbf-ov4 & $0.70 \pm 0.06 \mathrm{~b}$ & $0.78 \pm 0.04 \mathrm{~b}$ & & $0.50 \pm 0.00 \mathrm{~b}$ & $0.55 \pm 0.08 \mathrm{~b}$
\end{tabular}

Measurements were taken after 2 days on PDA medium. Controls are cultures without a previous T. harzianum growth. Values are means of three replicates with the corresponding standard deviations. For each column, values followed by different letter are significantly different $(P<0.05)$.

\section{Against Fusarium Wilt in Tomato Plants}

To evaluate the effects of pretreatment with $T$. harzianum T34 and the Thmbf1 overexpression on the development of Fusarium wilt disease caused by FO in 'Moneymaker' tomato plants, in vivo assays were performed using two T. harzianum application methods (Table 3). Supplementary Figure $\mathbf{S} 3$ shows the phenotype of tomato plants derived from T. harzianumtreated seeds and inoculated with FO. Typical symptoms of wilt disease were first observed 10 days after inoculation of FO with both T. harzianum application methods; the uninoculated tomato seedlings showed no symptoms. DI recorded at 21 days in FO-inoculated plants ranged from 50 to $56.2 \%$ and 48.5 to $53.7 \%$ for T. harzianum-treated seeds and T. harzianum-inoculated substrate, respectively. The lowest DI values were observed in $\mathrm{T} 34+\mathrm{FO}$ and Thmbf-CT + FO treatments in the substrate inoculation assay. No differences were detected between FO and Thmbf-CT + FO treatments. However, higher DI values were

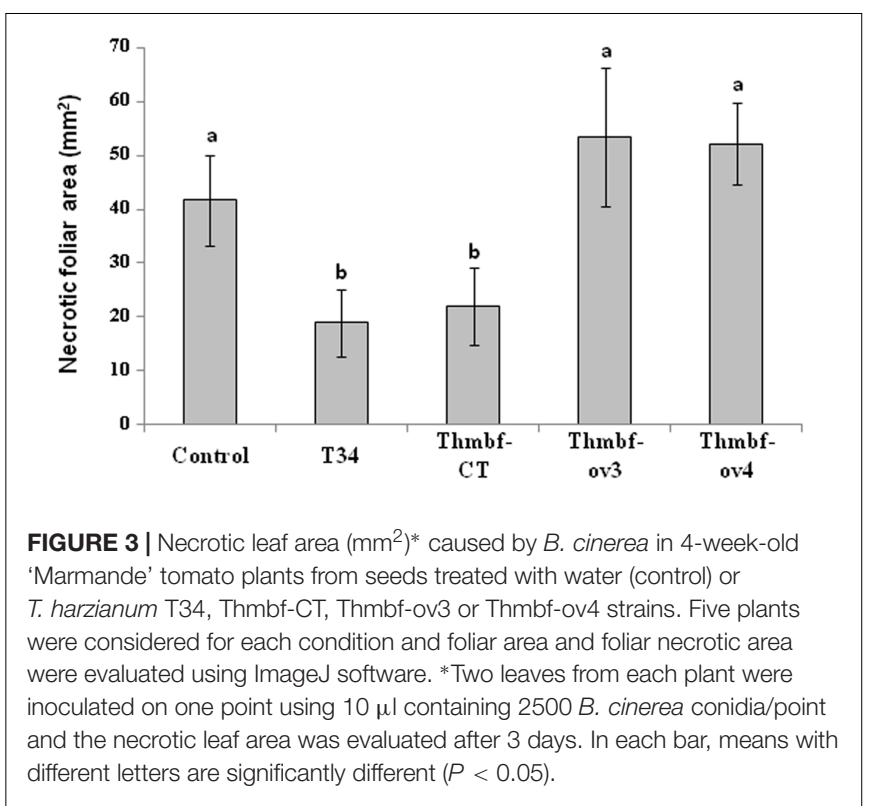

recorded for plants coming from T. harzianum-treated seeds, and later infected with FO, compared to those directly infected with FO. The highest DI values were observed in plants from Thmbfov3 + FO and Thmbf-ov4 + FO treatments, those with the Thmbf1-overexpressed transformants, in both assays. In addition, the lowest dry weight values were also observed in tomato plants previously seed-coated with a Thmbfl-overexpressing transformant and no significant differences were detected among plant dry weights from the treatments FO, T34 + FO and Thmbf$\mathrm{CT}+\mathrm{FO}$. Although the disease of tomato plants appears to be influenced by the method of inoculation of T. harzianum, taken all together, these results indicate that strain T34 did not show a biocontrol activity against FO in 'Moneymaker' plants, and that Thmbf1 overexpression in T34 reduced its antifungal activity against FO, leading to increased Fusarium wild disease. 
TABLE 3 | Effect of T. harzianum wild-type (T34), transformation control (Thmbf-CT) or Thmbf1 overexpressing transformants (Thmbf-ov3 and Thmbf-ov4) treatments on the development of disease caused by F. oxysporum f. sp. lycopersici (FO) in 'Moneymaker' tomato plants. Trichoderma strains were applied by inoculation of the substrate or by treatment of seeds.

\begin{tabular}{lcccc}
\hline & $\begin{array}{c}\text { Inoculated substrate } \\
\text { with T. harzianum }\end{array}$ & & \multicolumn{2}{c}{$\begin{array}{c}\text { T. harzianum- } \\
\text { treated seeds }\end{array}$} \\
\cline { 2 - 3 } \cline { 5 - 5 } & DI (\%) & & DI (\%) & Dry weight (g) \\
\hline Control & 0.0 & & 0.0 & $0.44 \pm 0.07 \mathrm{a}$ \\
FO & 52.5 & 50.0 & $0.19 \pm 0.03 \mathrm{~b}$ \\
T34 + FO & 50.0 & & 52.7 & $0.18 \pm 0.06 \mathrm{~b}$ \\
Thmbf-CT + FO & 48.5 & & 50.0 & $0.14 \pm 0.06 \mathrm{~b}$ \\
Thmbf-ov3 + FO & 53.7 & & 54.2 & $0.07 \pm 0.04 \mathrm{c}$ \\
Thmbf-ov4+FO & 53.7 & 56.2 & $0.10 \pm 0.05 \mathrm{c}$
\end{tabular}

Disease incidence (Dl), expressed in percentage, was determined after both application methods. Aboveground dry weights were also recorded for the assay T. harzianum treated-seeds. Controls are plants without a previous T. harzianum or FO treatment. *Values are means of ten replicates with the corresponding standard deviations, and those followed by different letter are significantly different $(P<0.05)$.

\section{DISCUSSION}

MBFs are highly conserved transcriptional coactivators present in Archaea and Eukarya (Takemaru et al., 1997; Tsuda et al., 2004; Suzuki et al., 2005), although not found in bacteria. This fact evidences the emergence of MBFs mediator proteins after the separation of the last archaeal common ancestor from the bacterial lineage (Forterre, 2013). MBF1 proteins control different physiological and/or developmental processes and, although few studies have been reported in fungi, they have also been related to virulence in B. bassiana and M. oryzae (Ying et al., 2014; Fan et al., 2017). In the present work, we explored the role of Thmbf1 gene in the antifungal activity of $T$. harzianum, since this species is one of the most cited as active ingredient in commercial biocontrol products (Lorito et al., 2010).

We identified the Thmbfl gene in a subtractive library prepared with cDNAs from T. harzianum T34 wild type and Thctf1 null mutant affected in the production of 6-PP derivatives (Rubio et al., 2009). These VOC are released by Trichoderma spp. as a component of their antifungal machinery (Mukherjee et al., 2013), and it has been described that a decreased production of 6-PP is correlated with loss of antifungal activity against pathogens such as Rhizoctonia solani, Sclerotinia sclerotiorum or F. oxysporum (Reithner et al., 2005; Rubio et al., 2009). The 6-PP is a major VOC biosynthesized by T. harzianum or T. atroviride species (Reino et al., 2008; Daoubi et al., 2009; Garnica-Vergara et al., 2016). It is able to induce growth promotion and reduce disease symptoms when applied at low concentrations to plant growth media or directly onto the leaves (Vinale et al., 2008). It has been demonstrated that Arabidopsis root responses to 6PP involves components of auxin transport as well as a master regulator of the ethylene-depending response pathway (GarnicaVergara et al., 2016).

We have isolated the Thmbf1 gene using a T34 genomic library (Lora et al., 1995). Since the frequency of homologous recombination in Trichoderma is very low and therefore null mutants are not easy to obtain in T. harzianum (Rosado et al., 2007; Rubio et al., 2009), the function of Thmbf1 was studied following an overexpression strategy. This approach limits comparison of the results with those from the three studies performed in filamentous fungi, in which a disruption strategy was followed (Schönig et al., 2009; Ying et al., 2014; Fan et al., 2017).

Southern blot data, showing one hybridization signal in both T34 and Thmbf-CT strains, as well as a single Thmbfl homolog identified in the publicly available Trichoderma genomes, indicate the existence of a single copy of this gene in the genus. These results are in agreement with those observed in other filamentous fungi, where a single $M B F$ ortholog has been described (Ying et al., 2014), whereas other organisms such as plants contain several MBF genes (Tsuda et al., 2004). Multiple additional copies of the gene were observed in three out of the four putative Thmbf1-transformants analyzed by Southern blotting (Supplementary Figure S1). However, there was no correlation between the expression levels and the additional gene copies. This lack of correlation has been also reported in Trichoderma transformant strains for genes such as chit $33, h s p 23$ or $h s p 70$ (Limón et al., 1999; Montero-Barrientos et al., 2007, 2008). The fact that Thmbf-ov1 did not show higher Thmbf1 transcript levels than the transformation control Thmbf-CT, after growing on rich or minimal medium, together with the absence of additional copies of the gene in its genome demonstrate that this is not an overexpressing transformant. When Thmbf-ov1 was further checked by PCR and grew in the presence of antibiotic, we could assess that this strain had not maintained the transformation cassette in its genome and the transforming DNA had been lost.

Since the expression of $c p c$ gene was not significantly modified in none of the five strains tested after growing in two different media, ThMBF1 does not appear to be linked to the master regulator $\mathrm{CPC} 1$ in T. harzianum. Our results are in contrast with the findings reported in yeast (Takemaru et al., 1998), but they are in agreement with those obtained by yeast two-hybrid assays carried out in F. fujikuroi (Schönig et al., 2009) and M. oryzae (Fan et al., 2017), where no interaction between MBF1 and CPC1 proteins was observed.

We used different in vitro assays to study the involvement of Thmbf1 in the antifungal activity of T. harzianum using two target phytopathogenic fungi. Thmbf-ov2 was the only transformant that showed a reduced antagonistic activity against FO and BC on dual culture assays (Supplementary Figure S2). However, it could be due to the low growth rate observed in this transformant. For this reason, we selected the strains Thmbf-ov3 and Thmbf-ov4 for further analysis. The fact that these Thmbf 1 overexpressing transformants showed lower antifungal activity against both pathogens when using a discontinuous medium, is indicative that VOC production is affected by Thmbf1. It is clear that a Thmbf1 overexpression in T. harzianum T34 modifies the communication mediated by VOC between the two physically separated fungi; it reduces the antifungal activity of the T34 strain. However, our results are not enough to conclude whether the observed differences are due to VOC produced by $T$. harzianum or there are also involved other compounds from the pathogen in that scenario. VOC are 
considered ideal info-chemicals that play important roles in the short- and long-distance interactions between physically separated microorganisms (Effmert et al., 2012; Schmidt et al., 2017). There is evidence that VOC play a role in T. harzianumF. oxyxporum confrontations, and that this pathogen induces the production of these type of compounds in the antagonist fungus (Zhang et al., 2014). The relationship between Thmbf1 and VOC in T. harzianum, deduced from assays performed in a discontinuous medium, should not be surprising since this gene has been identified in a SSH approach performed with a null mutant unable to produce several 6-PP derivatives (Rubio et al., 2009).

In addition to VOC, Thmbf1 could be involved in the production of low MW metabolites and/or enzymes with antifungal activity in $T$. harzianum since a significantly lower FO inhibition was detected for the two Thmbf1 overexpressing transformants in cellophane and dialysis membrane assays compared to those of wild type and the transformation control strains.

Trichoderma harzianum has demonstrated biocontrol potential in a wide range of crop plants against different pathogens (Monte, 2001; Harman et al., 2004; Lorito et al., 2010). In our study, according to the results obtained in the in vivo assays, T34 was able to reduce the lesions produced by $\mathrm{BC}$ in tomato plants but this ability was not observed in Thmbf1-overexpressing transformants. Considering that in this assay T. harzianum and BC were not in physical contact, the systemic defense mechanisms activated by strain T34 in the plant were not elicited enough when the Thmbf1 gene was overexpressed in the fungus. The beneficial effects of Trichoderma spp. regarding not only a direct antagonistic activity, but also the activation of systemic defense responses in the plant, depend on the strain, the genotype and age of the plant, the type of pathogen and the interaction conditions (Hermosa et al., 2012; Martínez-Medina et al., 2014). It is recognized that Trichoderma spp. are able to induce systemic resistance against necrotrophs like $\mathrm{BC}$ by signaling jasmonic acid (JA) and ethylene (ET)-dependent defense pathways (Shoresh et al., 2010). Moreover, they can activate the salicylic acid (SA)dependent defense responses (Rubio et al., 2014, 2017), which are crucial against biotrophic pathogens like FO (Glazebrook, 2005).

Despite of the wide host range shown by F. oxysporum, individual isolates are able to infect only one or a few plant species (Michielse and Rep, 2009). For in vivo assays we selected 'Moneymaker' tomato plants and used FO strain 4287 as the target pathogen because of its known virulence for this variety (Di Pietro and Roncero, 1998; Niño-Sánchez et al., 2016). Although T34 showed antifungal activity against FO in in vitro assays, no biocontrol efficacy was observed against this pathogen in two in vivo assays using different $T$. harzianum inoculation methods. Furthermore, tomato plants from the treatments with the Thmbf1-overexpressing transformants displayed the highest levels of Fusarium wilt disease. It is well known that the antifungal activity observed in in vitro assays for Trichoderma strains against different pathogens should not be extrapolated to other situations such as field or greenhouse conditions
(Hermosa et al., 2000). However, it has also been reported that Trichoderma spp. induce the above indicated JA/ETand SA-dependent defense responses in tomato plants (SalasMarina et al., 2011; Rubio et al., 2014), and that these fungi have demonstrated potential for suppressing Fusarium wilt development in tomato plants (Cotxarrera et al., 2002; Taghdi et al., 2015). These last works also demonstrated that the potential to suppress Fusarium wilt depends on the Trichoderma strain. Previous studies have shown that T34 is able to colonize the tomato rhizosphere (Morán-Diez et al., 2009; Samolski et al., 2012), and successful root colonization is considered a major prerequisite for the beneficial effects exerted by Trichoderma on plants (Lorito et al., 2010; Shoresh et al., 2010; Hermosa et al., 2012). Our greenhouse results indicate that T. harzianum T34 has the ability to reduce the lesion caused by BC in 'Marmande' tomato plants but it is not able to suppress the Fusarium wilt caused by FO in 'Moneymaker', at least under the assayed conditions. We have also found that the Thmbf1 overexpression in T. harzianum T34 negatively affected the biocontrol activity of this strain. Considering that reduced $B$. bassiana and $M$. oryzae virulence was observed in absence of MBF1 (Ying et al., 2014; Fan et al., 2017), and that Thmbf1 overexpressing transformants showed lower biocontrol potential than the wild type, it could be thought that adequate levels of MBF1 are needed to mediate the transcriptional pathways involved in the interactions of filamentous fungi with pathogens and plants.

In summary, we can conclude that the transcription coactivator MBF1 plays an important role in the biocontrol ability of T. harzianum, affecting the production and secretion of different antifungal compounds, and that the success of this fungus as a biocontrol agent depends on a suitable expression level of this fine adjustment regulator.

\section{AUTHOR CONTRIBUTIONS}

$\mathrm{MBR}$ and AP carried out in vitro assays. $\mathrm{RC}$ constructed the overexpressing plasmid and obtained transformants. SG made the subtractive cDNA library and the Southern blot. MBR and $\mathrm{RH}$ carried out greenhouse assays, prepared tables, figures and additional material. EM, RH, and MBR wrote the manuscript. $\mathrm{RH}$ designed and led the study. All authors have read and approved the final manuscript.

\section{ACKNOWLEDGMENTS}

This research project was funded by the Spanish Ministry of Economy and Competitiveness (Project no. AGL2015-70671-C2) and the Junta de Castilla y León (Project no. SA009U16).

\section{SUPPLEMENTARY MATERIAL}

The Supplementary Material for this article can be found online at: https://www.frontiersin.org/articles/10.3389/fmicb.2017. 02273/full\#supplementary-material 
FIGURE S1 | Southern blot analysis of wild-type (T34) and transformant strains. Genomic DNAs were Xhol- and BamHI-digested and the Thmbf1 CDNA was used as a probe. Lanes correspond to T. harzianum T34 (lane 1), Thmbf-CT (control transformant, lane 2), Thmbf-ov1 (lane 3), Thmbf-ov2 (lane 4), Thmbf-ov3 (lane 5) and Thmbf-ov4 (lane 6). EcoRl-Hindlll-digested $\lambda$ DNA was used as a marker and molecular sizes are indicated in kbp (lane 7).

FIGURE S2 | Dual cultures of strains T34, Thmbf-CT, Thmbf-ov1, Thmbf-ov2, Thmbf-ov3 and Thmbf-ov4 of T. harzianum and the pathogens F. oxyxporum (FO)

\section{REFERENCES}

Aravind, L., and Koonin, E. V. (1999). DNA-binding proteins and evolution of transcription regulation in the archaea. Nucleic Acids Res. 27, 4658-4670. doi: 10.1093/nar/27.23.4658

Arce, D. P., Tonón, C., Zanetti, M. E., Godoy, A. V., Hirose, S., and Casalongué, C. A. (2006). The potato transcriptional co-activator StMBF1 is up-regulated in response to oxidative stress and interacts with the TATA-box binding protein. J. Biochem. Mol. Biol. 39, 355-360. doi: 10.5483/BMBRep.2006. 39.4.355

Aro, N., Ilmén, M., Saloheimo, A., and Penttilä, M. (2003). ACE1 of Trichoderma reesei is a repressor of cellulase and xylanase expression. Appl. Environ. Microbiol. 69, 56-65. doi: 10.1128/AEM.69.1.56-65.2003

Brendel, C., Gelman, L., and Auwerx, J. (2002). Multiprotein bridging factor-1 (MBF-1) is a cofactor for nuclear receptors that regulate lipid metabolism. Mol. Endocrinol. 16, 1367-1377. doi: 10.1210/mend.16.6.0843

Cardoza, R. E., Vizcaíno, J. A., Hermosa, M. R., Monte, E., and Gutiérrez, S. (2006a). A comparison of the phenotypic and genetic stability of recombinant Trichoderma spp. generated by protoplast- and Agrobacterium-mediated transformation. J. Microbiol. 44, 383-395.

Cardoza, R. E., Vizcaíno, J. A., Hermosa, M. R., Sousa, S., González, F. J., Llobell, A., et al. (2006b). Cloning and characterization of the erg1 gene of Trichoderma harzianum: effect of the erg1 silencing on ergosterol biosynthesis and resistance to terbinafine. Fungal Genet. Biol. 43, 269-283. doi: 10.1016/j.fgb.2005.11.002

Casas-Flores, S., Rios-Momberg, M., Bibbins, M., Ponce-Noyola, P., and Herrera-Estrella, A. (2004). BLR-1 and BLR-2, key regulatory elements of photoconidiation and mycelial growth in Trichoderma atroviride. Microbiology 150, 3561-3569. doi: 10.1099/mic.0.27346-0

Cotxarrera, L., Trillas-Gay, M. I., Steinberg, C., and Alabouvette, C. (2002). Use of sewage sludge compost and Trichoderma asperellum isolates to suppress Fusarium wilt of tomato. Soil Biol. Biochem. 34, 467-476. doi: 10.1016/S00380717(01)00205-X

Daoubi, M., Pinedo-Rivilla, C., Rubio, M. B., Hermosa, R., Monte, E., Aleu, J., et al. (2009). Hemisynthesis and absolute configuration of novel 6-pentyl-2Hpyran-2-one derivatives from Trichoderma spp. Tetrahedron 65, 4834-4840. doi: 10.1016/j.tet.2009.04.051

Di Pietro, A., and Roncero, M. I. G. (1998). Cloning, expression, and role in pathogenicity of $p g 1$ encoding the major extracellular endopolygalacturonase of the vascular wilt pathogen Fusarium oxysporum. Mol. Plant Microbe Interact. 11, 91-98. doi: 10.1094/MPMI.1998.11.2.91

Diatchenko, L., Lau, Y. F., Campbell, A. P., Chenchik, A., Mogadam, F., Huang, B., et al. (1996). Suppression subtractive hybridization: a method for generating differentially regulated or tissue-specific cDNA probes and libraries. Proc. Natl. Acad. Sci. U.S.A. 93, 6025-6030. doi: 10.1073/pnas.93.12.6025

Druzhinina, I. S., Seidl-Seiboth, V., Herrera-Estrella, A., Horwitz, B. A., Kenerley, C. M., Monte, E., et al. (2011). Trichoderma: the genomics of opportunistic success. Nat. Rev. Microbiol. 9, 749-759. doi: 10.1038/nrmicro2637

Effmert, U., Kalderas, J., Warnke, R., and Piechulla, B. (2012). Volatile mediated interactions between bacteria and fungi in the soil. J. Chem. Ecol. 38, 665-703. doi: 10.1007/s10886-012-0135-5

Fan, G., Zhang, K., Huang, H., Zhang, H., Zhao, A., Chen, I., et al. (2017). Multiprotein-bridging factor 1 regulates vegetative growth, osmotic stress, and virulence in Magnaporthe oryzae. Curr. Genet. 63, 293-309. doi: 10.1007/ s00294-016-0636-9

Forterre, P. (2013). The common ancestor of Archaea and Eukarya was not an archaeon. Archaea 2013:372396. doi: 10.1155/2013/372396
(A) and B. cinerea (BC) (B) on continuous PDA medium. Plates only with the pathogen were used as controls. All plates were incubated at $28^{\circ} \mathrm{C}$ for 10 days.

FIGURE S3 | Phenotype of 'Moneymaker' tomato plants derived from T. harzianum-treated seeds and inoculated with FO. The wild-type T34, the transformation control (Thmbf-CT), and the Thmbf1 overexpressing transformants (Thmbf-ov3 and Thmbf-ov4) were applied as T. harzianum strains. Plants without T. harzianum or FO treatment were used as controls. Photographs were taken 3 weeks after $\mathrm{FO}$ inoculations.

Fu, K., Fan, L., Gao, S., and Chen, J. (2012). Tmac1, a transcription factor which regulated high affinity copper transport in Trichoderma reesei. Microbiol. Res. 167, 536-543. doi: 10.1016/j.micres.2012.02.002

Garnica-Vergara, A., Barrera-Ortiz, S., Muñoz-Parra, E., Raya-González, J., Méndez-Bravo, A., Macías-Rodríguez, L., et al. (2016). The volatile 6-pentyl2H-pyran-2-one from Trichoderma atroviride regulates Arabidopsis thaliana root morphogenesis via auxin signaling and ethylene insensitive 2 functioning. New Phytol. 209, 1496-1512. doi: 10.1111/nph.13725

Glazebrook, J. (2005). Contrasting mechanisms of defense against biotrophic and necrotrophic pathogens. Annu. Rev. Phytopathol. 43, 205-227. doi: 10.1146/ annurev.phyto.43.040204.135923

Godoy, A. V., Zanetti, M. E., San Segundo, B., and Casalongué, C. A. (2001). Identification of a putative Solanum tuberosum transcriptional coactivator up-regulated in potato tubers by Fusarium solani f. sp. eumartii infection and wounding. Physiol. Plant. 112, 217-222. doi: 10.1034/j.1399-3054.2001. 1120210.x

Gruber, S., and Zeilinger, S. (2014). The transcription factor Ste12 mediates the regulatory role of the Tmk1 MAP kinase in mycoparasitism and vegetative hyphal fusion in the filamentous fungus Trichoderma atroviride. PLOS ONE 9:e111636. doi: 10.1371/journal.pone.0111636

Gutiérrez, S., Velasco, J., Marcos, A. T., Fernández, F. J., Fierro, F., Barredo, J. L., et al. (1997). Expression of the cefG gene is limiting for cephalosporin biosynthesis in Acremonium chrysogenum. Appl. Microbiol. Biotechnol. 48, 606-614. doi: 10.1007/s002530051103

Harman, G. E., Howell, C. R., Viterbo, A., Chet, I., and Lorito, M. (2004). Trichoderma species opportunistic, avirulent plant symbionts. Nat. Rev. Microbiol. 2, 43-56. doi: 10.1038/nrmicro797

Hermosa, M. R., Grondona, I., Iturriaga, E. A., Diaz-Mínguez, J. M., Castro, C., Monte, E., et al. (2000). Molecular characterization and identification of biocontrol isolates of Trichoderma spp. Appl. Environ. Microbiol. 66, 1890-1898. doi: 10.1128/AEM.66.5.1890-1898.2000

Hermosa, R., Viterbo, A., Chet, I., and Monte, E. (2012). Plant-beneficial effects of Trichoderma and of its genes. Microbiology 158, 17-25. doi: 10.1099/mic.0. 052274-0

Hinnebusch, A. G., and Natarajan, K. (2002). Gcn4p, a master regulator of gene expression, is controlled at multiple levels by diverse signals of starvation and stress. Eukaryot. Cell 1, 22-32. doi: 10.1128/EC.01.1.2232.2002

Kabe, Y., Yamada, J., Uga, H., Yamaguchi, Y., Wada, T., and Handa, H. (2005). NF-Y is essential for the recruitment of RNA polymerase II and inducible transcription of several CCAAT box-containing genes. Mol. Cell. Biol. 25, 512-522. doi: 10.1128/MCB.25.1.512-522.2005

Li, F. Q., Ueda, H., and Hirose, H. (1994). Mediators of activation of fushi tarazu gene transcription by BmFTZ-F1. Mol. Cell. Biol. 14, 3013-3021. doi: 10.1128/ MCB.14.5.3013

Limón, M. C., Pintor-Toro, J. A., and Benítez, T. (1999). Increased antifungal activity of Trichoderma harzianum transformants that overexpress a 33-kDa chitinase. Phytopathology 107, 7-12. doi: 10.1094/PHYTO.1999. 89.3.254

Liu, Q. X., Jindra, M., Ueda, H., Hiromi, Y., and Hirose, S. (2003). Drosophila $\mathrm{MBF} 1$ is a co-activator for tracheae defective and contributes to the formation of tracheal and nervous systems. Development 130, 719-728. doi: 10.1242/dev. 00297

Livak, K. J., and Schmittgen, T. D. (2001). Analysis of relative gene expression data using real-time quantitative PCR and the $2^{-\Delta \Delta C_{\mathrm{T}}}$ method. Methods 25, 402-408. doi: 10.1006/meth.2001.1262 
Lora, J. M., De la Cruz, J., Llobell, A., Benítez, T., and Pintor-Toro, J. A. (1995). Molecular characterization and heterologous expression of an endo-beta-1,6glucanase gene from the mycoparasitic fungus Trichoderma harzianum. Mol. Gen. Genet. 247, 639-645. doi: 10.1007/BF00290356

Lorito, M., Woo, S. L., Harman, G. E., and Monte, E. (2010). Translational research on Trichoderma: from 'omics to the field. Ann. Rev. Phytopathol. 48, 395-417. doi: 10.1146/annurev-phyto-073009-114314

Martínez-Medina, A., Alguacil, M. M., Pascual, J. A., and Van Wees, S. C. M. (2014). Phytohormone profiles induced by Trichoderma isolates correspond with their biocontrol and plant growth-promoting activity on melon plants. J. Chem. Ecol. 40, 804-815. doi: 10.1007/s10886-014-0478-1

Michielse, C. B., and Rep, M. (2009). Pathogen profile update: Fusarium oxysporum. Mol. Plant Pathol. 10, 311-324. doi: 10.1111/j.1364-3703.2009. 00538.x

Miotto, B., and Struhl, K. (2006). Differential gene regulation by selective association of transcriptional co-activators and bZIP DNA-binding domains. Mol. Cell. Biol. 26, 5969-5982. doi: 10.1128/MCB.00696-06

Monte, E. (2001). Understanding Trichoderma: between biotechnology and microbial ecology. Int. Microbiol. 4, 1-4. doi: 10.1007/s101230100001

Montero-Barrientos, M., Cardoza, R. E., Gutiérrez, S., Monte, E., and Hermosa, R. (2007). The heterologous overexpression of hsp23, a small heat-shock protein gene from Trichoderma virens, confers thermotolerance to T. harzianum. Curr. Genet. 52, 45-53. doi: 10.1007/s00294-007-0140-3

Montero-Barrientos, M., Hermosa, R., Cardoza, R. E., Gutiérrez, S., Nicolás, C., and Monte, E. (2010). Transgenic expression of the Trichoderma harzianum hsp70 gene increases Arabidopsis resistance to heat and other abiotic stresses. J. Plant Physiol. 167, 659-665. doi: 10.1016/j.jplph.2009.11.012

Montero-Barrientos, M., Hermosa, R., Nicolás, C., Cardoza, R. E., Gutiérrez, S., Monte, E., et al. (2008). Overexpression of a Trichoderma HSP70 gene increases fungal resistance to heat and other abiotic stresses. Fungal Gent. Biol. 45, 1506-1513. doi: 10.1016/j.fgb.2008.09.003

Morán-Diez, E., Hermosa, R., Ambrosino, P., Cardoza, R. E., Gutiérrez, S., Lorito, M., et al. (2009). The ThPG1 endopolygalaturonase is required for the Trichoderma harzianum-plant beneficial interaction. Mol. Plant Microbe Int. 22, 1021-1031. doi: 10.1094/MPMI-22-8-1021

Mukherjee, P. K., Horwitz, B. A., Herrera-Estrella, A., Schmoll, M., and Kenerley, C. M. (2013). Trichoderma research in the genomic era. Annu. Rev. Phytopathol. 51, 105-129. doi: 10.1146/annurev-phyto-082712-102353

Niño-Sánchez, J., Casado-del Castillo, V., Tello, V., de Vega-Bartol, J. J., Ramos, B., Sukno, S. A., et al. (2016). The FTF gene family regulates virulence and expression of SIX effectors in Fusarium oxysporum. Mol. Plant Pathol. 17, 1124-1129. doi: 10.1111/mpp.12373

Paluh, J. L., Orbach, M. J., Legerton, T. L., and Yanofsky, C. (1988). The crosspathway control gene of Neurospora crassa, cpc-1, encodes a protein similar to GNC4 of yeast and the DNA-binding domain of the oncogene v-jun-encoded protein. Proc. Natl. Acad. Sci. U.S.A. 85, 3728-3372. doi: 10.1073/pnas.85.11. 3728

Penttilä, M., Nevalainen, H., Ratto, M., Salminen, E., and Knowles, J. (1987). A versatile transformation system for the cellulolytic filamentous fungus Trichoderma reesei. Gene 61, 155-164. doi: 10.1016/0378-1119(87) 90110-7

Pérez, E., Rubio, M. B., Cardoza, R. E., Gutiérrez, S., Bettiol, W., Monte, E., et al. (2015). The importance of chorismate mutase in the biocontrol potential of Trichoderma parareesei. Front. Microbiol. 6:1181. doi: 10.3389/fmicb.2015. 01181

Punt, P. J., Oliver, R. P., Dingemanse, M. A., Pouwels, P. H., and van den Hondel, C. A. (1987). Transformation of Aspergillus based on the hygromycin B resistance marker from Escherichia coli. Gene 56, 117-124. doi: 10.1016/03781119(87)90164-8

Reino, J. L., Guerrero, R. F., Hernández-Galán, R., and Collado, I. G. (2008). Secondary metabolites from species of the biocontrol agent Trichoderma. Phytochem. Rev. 7, 89-123. doi: 10.1007/s11101-006-9032-2

Reithner, B., Brunner, K., Schuhmacher, R., Peissl, I., Seidl, V., Krska, R., et al. (2005). The G protein alpha subunit Tga1 of Trichoderma atroviride is involved in chitinase formation and differential production of antifungal metabolites. Fungal Genet. Biol. 42, 749-760. doi: 10.1128/EC.1.4.594-605.2002

Rosado, I. V., Rey, M., Codón, A. C., Govantes, J., Moreno-Mateos, M. A., and Benítez, T. (2007). QID74 cell wall protein of Trichoderma harzianum is involved in cell protection and adherence to hydrophobic surfaces. Fungal Genet. Biol. 44, 950-964. doi: 10.1016/j.fgb.2007.01.001

Rubio, M. B., Hermosa, R., Reino, J. L., Collado, I. G., and Monte, E. (2009). Thctf1 transcription factor of Trichoderma harzianum is involved in 6-pentyl- $2 \mathrm{H}-$ pyran-2-one production and antifungal activity. Fungal Genet. Biol. 46, 17-27. doi: 10.1016/j.fgb.2008.10.008

Rubio, M. B., Hermosa, R., Vicente, R., Gómez-Acosta, F. A., Morcuende, R., Monte, E., et al. (2017). The combination of Trichoderma harzianum and chemical fertilization leads to the deregulation of phytohormone networking, preventing the adaptative responses of tomato plants to salt stress. Front. Plant Sci. 8:294. doi: 10.3389/fpls.2017.00294

Rubio, M. B., Quijada, N. M., Pérez, E., Domínguez, S., Monte, E., and Hermosa, R. (2014). Identifying beneficial qualities of Trichoderma parareesei for plants. Appl. Environ. Microbiol. 80, 1864-1873. doi: 10.1128/AEM.03375-13

Salas-Marina, M. A., Silva-Flores, M. A., Uresti-Rivera, E. E., Castro-Longoria, E., Herrera-Estrella, A., and Casas-Flores, S. (2011). Colonization of Arabidopsis roots by Trichoderma atroviride promotes growth and enhances systemic disease resistance through jasmonic acid/ethylene and salicylic acid pathways. Eur. J. Plant Pathol. 131, 15-26. doi: 10.1007/s10658-011-9782-6

Samolski, I., Rincón, A. M., Pinzón, L. M., Viterbo, A., and Monte, E. (2012). The quid74 gene from Trichoderma harzianum has a role in root architecture and plant biofertilization. Microbiology 158, 129-138. doi: 10.1099/mic.0.0 53140-0

Schmidt, R., de Jager, V., Zühlke, D., Wolff, C., Bernhardt, J., Cankar, K., et al. (2017). Fungal volatile compounds induce production of the secondary metabolite Sodorifen in Serratia plymuthica PRI-2C. Sci. Rep. 7, 862. doi: 10.1038/s41598-017-00893-3

Schönig, B., Brown, D. W., Oeser, B., and Tudzynski, B. (2008). Cross-species hybridization with Fusarium verticillioides microarrays reveals new insights into Fusarium fujikuroi nitrogen regulation and the role of AreA and NMR. Eukaryot. Cell 7, 1831-1846. doi: 10.1128/EC.00130-08

Schönig, B., Vogel, S., and Tudzynski, B. (2009). Cpc1 mediates cross-pathway control independently of Mbf1 in Fusarium fujikuroi. Fungal Genet. Biol. 46, 898-908. doi: 10.1016/j.fgb.2009.08.003

Shoresh, M., Harman, G. E., and Mastouri, F. (2010). Induced systemic resistance and plant responses to fungal biocontrol agents. Annu. Rev. Phytopathol. 48, 21-43. doi: 10.1146/annurev-phyto-073009-114450

Song, W., Zhou, L., Yang, C., Cao, X., Zhang, L., and Liu, X. (2004). Tomato Fusarium wilt and its chemical control strategies in hydroponic system. Crop Prot. 23, 243-247. doi: 10.1016/j.cropro.2003.08.007

Stricker, A. R., Grosstessner-Hain, K., Würleiner, E., and Mach, R. L. (2006). Xyr1 (xylanase regulator 1) regulates both the hydrolytic enzyme system and D-xylose metabolism in Hypocrea jecorina. Eukaryot. Cell 5, 2128-2137. doi: 10.1128/EC.00211-06

Suzuki, N., Bajad, S., Shuman, J., Shulaev, V., and Mittler, R. (2008). The transcriptional co-activator MBF1c is a key regulator of thermotolerance in Arabidopsis thaliana. J. Biol. Chem. 283, 9269-9275. doi: 10.1074/jbc. M709187200

Suzuki, N., Rizhsky, L., Liang, H., Shuman, J., Shulaev, V., and Mittler, R. (2005). Enhanced tolerance to environmental stress in transgenic plants expressing the transcriptional coactivator multiprotein bridging factor 1c. Plant Physiol. 139, 1313-1322. doi: 10.1104/pp.105.070110

Taghdi, Y., Hermosa, R., Domínguez, S., Rubio, M. B., Essalmani, H., Nicolás, C., et al. (2015). Effectiveness of composts and Trichoderma strains in the control of tomato Fusarium wilt. Phytopathol. Mediterr. 54, 232-240. doi: 10.14601/ Phytopathol_Mediterr-15226

Takemaru, K., Harashima, S., Ueda, H., and Hirose, S. (1998). Yeast coactivator MBF1 mediates GCN4-dependent transcriptional activation. Mol. Cell. Biol. 18, 4971-4976. doi: 10.1128/MCB.18.9.4971

Takemaru, K., Li, F., Ueda, H., and Hirose, S. (1997). Multiprotein bridging factor 1 $(\mathrm{MBF} 1)$ is an evolutionarily conserved transcriptional coactivator that connects a regulatory factor and TATA element-binding protein. Proc. Natl. Acad. Sci. U.S.A. 94, 7251-7256. doi: 10.1073/pnas.94.14.7251

Tijerino, M., Cardoza, R. E., Moraga, J., Malmierca, M. G., Vicente, F., Aleu, J., et al. (2011). Overexpression of the trichodiene synthase gene tri5 increases trichodermin production and antimicrobial activity in Trichoderma brevicompactum. Fungal Genet. Biol. 48, 285-296. doi: 10.1016/j.fgb.2010. 11.012 
Tsuda, K., Tsuji, T., Hirose, S., and Yamazaki, K. (2004). Three Arabidopsis MBF1 homologs with distinct expression profiles play roles as transcriptional co-activators. Plant Cell Physiol. 45, 225-231. doi: 10.1093/pcp/pch017

Vinale, F., Sivasithamparam, K., Ghisalberti, E. L., Marra, R., Barbetti, M. J., Li, H., et al. (2008). A novel role for Trichoderma secondary metabolites in the interactions with plants. Physiol. Mol. Plant Pathol. 72, 80-86. doi: 10.1016/j. pmpp.2008.05.005

Ying, S. H., Ji, X. P., Wang, X. X., Feng, M. G., and Keyhani, N. O. (2014). The transcriptional co-activator multiprotein bridding factor 1 from the fungal insect pathogen, Beauveria bassiana, mediates regulation of hyphal morphogenesis, stress tolerance and virulence. Environ. Microbiol. 16, 1879-1897. doi: 10.1111/1462-2920.12450

Zhang, F., Yang, X., Ran, W., and Shen, Q. (2014). Fusarium oxyxporum induces the production of proteins and volatile organic compounds by Trichoderma harzianum T-E5. FEMS Microbiol. Lett. 359, 116-123. doi: 10.1111/1574-6968. 12582

Conflict of Interest Statement: The authors declare that the research was conducted in the absence of any commercial or financial relationships that could be construed as a potential conflict of interest.

Copyright (๐ 2017 Rubio, Pardal, Cardoza, Gutiérrez, Monte and Hermosa. This is an open-access article distributed under the terms of the Creative Commons Attribution License (CC BY). The use, distribution or reproduction in other forums is permitted, provided the original author(s) or licensor are credited and that the original publication in this journal is cited, in accordance with accepted academic practice. No use, distribution or reproduction is permitted which does not comply with these terms. 